\title{
Gluon Distributions in Real and Virtual Photon and the Photon Structure Function
}

\author{
B.L.Ioffe \\ Institute of Theoretical and Experimental Physics \\ B.Cheremushkinskaya 25, 117259, Moscow, Russia \\ (e-mail: ioffe@vxitep.itep.ru) \\ FAX 7(095) 8839605 \\ and \\ A.Oganesian \\ Yerevan Physics Institute \\ Alikhanian Brothers St.,2, 375036, Yerevan, Armenia \\ (e-mail: armen@vxitep.itep.ru)
}

\begin{abstract}
Gluon distributions in real and virtual photons are calculated using evolution equations in the NLO approximation. The quark distributions in the photon determined on the basis of the QCD sum rule approach in ref.[1] are taken as an input. It is shown that gluon distribution in the photon can be reliably determined up to $x=0.03 \div 0.05$ much lower than the corresponding values in the case of quark distributions. Two variants of the calculations are considered: (1) it is assumed that there are no intrinsic gluons in the photon at some low normalization point $Q^{2}=Q_{0}^{2} \sim 1 \mathrm{GeV}^{2} ;(2)$ it is assumed that gluonic content of the photon at low $Q_{0}^{2}$ is described by gluonic content of vector mesons $\rho, \omega, \varphi$. The gluon distributions in these two variants appear to be different. This fact permits one to clarify the origin of nonperturbative gluonic content of the photon by comparing the results with experiment.

Structure functions $F_{2}(x)$ for real and virual photon are calculated and it is shown that in the region $x \geq 0.2$ where QCD approach is valid, there is a good agreement with experiment.
\end{abstract}




\section{Introduction}

In paper [1] the structure functions of real and virtual photons were calculated. The hadronic part of the photon structure function was calculated in a modelless way on the basis of the QCD sum rules applying to structure functions $[2,3]$. In this aspect the calculation made in [1] differs from earlier considerations of the same problem [4-7]. The idea of the ref.[1] consideration was the following. First, the structure function of the virtual photon (photon-target) was studied under conditions when photon virtuality $p^{2}<0$ and $\left|p^{2}\right|>>R_{c}^{-2}, R_{c}$ - is the confiniment radius, but at the same time $\left|p^{2}\right|<<Q^{2}=-q^{2}$, where $q$ - is the photon projectile momentum. In expansion in power in $p^{2} / q^{2}$ only terms of the first nonvanishing order were taken into account - the terms of the lowest twist -2 . The operator product expansion (OPE) over $1 / p^{2}$ was performed and the first and second order terms were taken into account. The contribution of the latter corresponds to interaction of quarks with vacuum gluonic fields and leads to appearance of terms proportional to gluonic condensate in the structure function of the virtual photon. Thus, the structure function of the virtual photon $F_{2}\left(s, p^{2}\right), s=(p+q)^{2}$ was constructed as a series in $1 / p^{2}$. On the other hand, using analytical properties of $F_{2}\left(s, p^{2}\right)$ as functions of $p^{2}$ by means of dispersion relations in $p^{2}$ at fixed $s F_{2}\left(s, p^{2}\right)$ was presented through contributions of physical states modelled as contributions of vector mesons $(\rho, \varphi, \omega)$ and continuum. By identifying both of these representations the structure function of the virtual photon including its hadronic part was found. At such approach the structure function possesses correct analytical properties in $p^{2}$ and has no fictitious singularties at $p^{2}=0$ inherent to bare diagram of Fig.1 (for massless quarks).

The result of the calcultions of the transverse photon structure function is [1]:

$$
\begin{gathered}
\left(\frac{3 \alpha}{\pi}\right)^{-1} F_{2}^{T}\left(x, p^{2}, Q^{2}\right)=x \sum_{q} e_{q}^{4}\left\{\left[2+\kappa(x)\left(\ln \frac{Q^{2}}{x^{2}\left(s_{0}-p^{2}\right)}-3+\right.\right.\right. \\
\left.\left.\left.+\frac{p^{2}}{s_{0}-p^{2}}\right)\right]+\frac{1}{2} \frac{s_{0}^{2}}{\left(p^{2}-m_{V, q}^{2}\right)^{2}}\left[\kappa(x)-\frac{8 \pi^{2}}{27 s_{0}^{2} x^{2}}<0\left|\frac{\alpha_{s}}{\pi} G_{\mu \nu}^{2}\right| 0>\right]\right\},
\end{gathered}
$$

where $x=Q^{2} / 2 \nu$ is the Bjorken variable, $\kappa(x)=x^{2}+(1-x)^{2}, s_{0}=1.5 \mathrm{GeV}^{2}$ is the continuum threshold, $m_{V, q}$ are the vector meson masses, $\rho$ and $\omega$ for 
$q=u, d$ and $\varphi$ for $q=s$. (We restrict ourselves to consideration of three flavours).

As has been shown in [1] and as is seen from (1) the first term in square brackets in the right-hand side of (1) corresponds to the continuum contribution in dispersion representation in $p^{2}$ while the second to the vector-meson contribution. Applying duality considerations we arrive at the conclusion that the first term in the right-hand side of (1) can be considered as a photon perturbative contribution into $F_{2}$, and the second term as hadronic part of the structure function $F_{2}$. Thereby, we unambiquously select from the diagram of Fig.1 the soft and collinear quark contribution which can referred to quark sea distribution in photon but not to direct contribution of photonic operators in OPE.

At $x>0.2$ the gluonic condensate contribution in (1) is less than $15 \%$ even at $p^{2}=0$. This allows one (at these values of $x$ ) to extrapolate (1) to the point $p^{2}=0$ and to get structure function of the real photon.

An essential disadvantage of the discussed method of the structure functions calculation $[2,3,1]$ is that it is inapplicable at small $x$ and $x$ close to 1 . As is seen from (1), the correction term of OPE proportional to gluonic condensate fastly increases with $x$ decreasing, i.e. the series in $1 / p^{2}$ diverges at small $x$. This is caused by the fact that in the imaginary part of the forward $\gamma-\gamma$ scattering amplitude - the diagrams of Fig. 1 - the quark virtuality at vertical quark lines $k^{2} \sim x p^{2}$ for massless quarks. Thus, at small $x$ quarks appear to be in near the mass shell, i.e., in the nonperturbative region. At $x$ close to 1 , the situation is similar [3]. As was shown in [1], eq.1 is true for the real photon at $0.2<x<0.7$. With $\left|p^{2}\right|$ increasing the applicability region of (1) expands.

In expression (1) for the structure function $F_{2}\left(x, p^{2}, Q^{2}\right)$ the evolution of $F_{2}\left(x, p^{2}, Q^{2}\right)$ with $Q^{2}$ was not accounted, because for the solution of the evolution equations it is necessary to know $F_{2}$ throughout the whole interval of $x$. That it why one may expect that (1) is true at intermadiate values $Q^{2} \sim 5-10 \mathrm{GeV}^{2}$, where the role of evolution is still inessential. (When comparing with the experiment it turned out that (1) well describes experiment even for $Q^{2} \approx 20 \mathrm{GeV}^{2}$ ).

In this paper we will try to partially correct this disadvantage, to take into account the evolution equations and to calculate gluonic distribution in the real and virtual photon. The calculation of gluonic distribution is preferable by virtue of our approach because gluons are produced by quarks and gluon 
energies are essentially smaller than quark energies. This allows us to hope (and is confirmed by calculation) that basing on the known quark distributions in the photon at $x>0.2$ we shall be able to find gluonic distribution at noticeably smaller $x$. (The $x>0.8$ region weakly affects gluonic distribution at $x<0.5)$. The interest to gluonic distribution in the photon is also connected with the proposal to measure it on HERA accelerator [8].

Calculations will be performed in the leading (LO) and next to leading (NLO) approximation. We consider two variants of the boundary conditions.

The first variant is based on an assumption that all gluonic distribution in the photon stems only from their emission by quarks and that the photon has no intrinsic gluons of nonperturbative origin. I.e., we will believe that at some low normalization point $Q^{2}=Q_{0}^{2}$ the gluonic distribution (as well as the sea quark distribution - we determine them in Sec.3) vanishes. This idea is close to the forwarded earlier analogous idea on the origin of gluons and sea quarks in hadrons (see, e.g. [9]). It seems that in the case of a photon, espesially of a virtual one, such an idea has much more rights for existence than in the case of hadrons. Let us once more emphasize that the main difference between this consideration and the previous ones (see, e.g. [10-20]) is that the hadronic part of the structure function of a photon at $Q^{2}=Q_{0}^{2}$ is taken from [1] and the point-like photon is separated from its hadronic components in a way as it was explained above.

The second variant of the boundary conditions is also related to the results of [1]. The second (hadronic) term in eq.(1) corresponds to the contribution of vector meson, i.e. to transition of a photon into vector meson with subsequent scattering of the projectile virtual photon on this vector meson (see Fig.2). Therefore one may speak about gluonic sea of the photon which corresponds to the vector meson intrinsic gluons (naturally, with a corresponding normalization).

As follows from our results, the gluonic distribution appears to be essentially different in these two variants. Thus, comparison of gluonic distribution with experiment would clarify hadronic structure of photon.

In Sec. 2 we discuss the evolution equations in the NLO approximation. In Sec.3 we will find their solution and discuss the results obtained. 


\section{Evolution Equations. Partial Solution of Inhomoqeneus Equation.}

As has been originally shown by Witten [10], evolution of the photon structure function differs from the standard evolution of the structure functions of hadrons. The reason for this is that in the photon case there exists an additional mechanism of direct (point-like) production of quarks from photon (as well as of gluons in the next order in $\alpha_{s}$ ). This mechanism results in logarithmic increasing of the structure function with $Q^{2}$ unlike evolution of hadronic structure functions where quarks and gluons may be produced only from quarks or gluons. Formally, in the OPE technique the moment expansion of the photon structure function has the form [10]

$$
\begin{gathered}
F_{2}^{n}\left(Q^{2}\right) \equiv \int_{0}^{1} d x \cdot x^{n-2} F_{2}\left(x, Q^{2}\right)=\sum_{i=N S, S, G} \hat{C}_{n}^{i}\left(Q^{2} / \mu^{2}, g\left(\mu^{2}\right)\right)<\gamma\left|\hat{O}_{n}^{i}\right| \gamma>+ \\
+C_{n}^{\gamma}\left(Q^{2} / \mu^{2}, g\left(\mu^{2}\right)\right)<\gamma\left|O_{n}^{\gamma}\right| \gamma>
\end{gathered}
$$

Here $\hat{O}_{n}^{i}=\left(O_{n}^{N S}, O_{n}^{S}, O_{n}^{G}\right) \equiv \hat{O}_{n}$, correspondingly, nonsinglet and singlet quark and gluon operators of spin $n$ and twist-2 and $O_{n}^{\gamma}$ are the photon operators (see e.g.[11]), $\hat{C}_{n}^{i}\left(Q^{2} / \mu^{2}, g^{2}(\mu)\right)$ is the column of Wilson coefficients

$$
\hat{C}_{n}^{i}\left(Q^{2} / \mu^{2}, g(\mu)\right)=\left[\begin{array}{l}
C_{n}^{N S}\left(Q^{2} / \mu^{2}, g(\mu)\right) \\
C_{n}^{S}\left(Q^{2} / \mu^{2}, g(\mu)\right) \\
C_{n}^{G}\left(Q^{2} / \mu^{2}, g(\mu)\right)
\end{array}\right] \equiv \hat{C}_{n}\left(Q^{2} / \mu^{2}, g(\mu)\right)
$$

As was noted in [10], the second term in (2) for the case of hadrons would be the $O(\alpha)$ correction, since $C_{n}^{\gamma}\left(Q^{2} / \mu^{2}, g(\mu) \sim O(\alpha)\right.$. For the case of photons this term should be also taken into account because matrix element $<\gamma \mid$ $\hat{O}_{n}^{i} \mid \gamma>$ in the first term of (2) is also of order $\alpha\left(<\gamma\left|O_{n}^{\gamma}\right| \gamma>\right.$ is, naturally of order 1).

Thus, the renormalization group equation for $C_{n}$ takes the form [10]:

$$
\left(\mu \frac{\partial}{\partial \mu}+\beta(y) \frac{\partial}{\partial y}\right)\left[\begin{array}{l}
\hat{C}_{n} \\
C_{n}^{\gamma}
\end{array}\right]=\left(\begin{array}{ll}
\hat{\gamma}_{n} & O \\
\mathbf{K}_{n} & O
\end{array}\right)\left[\begin{array}{l}
\hat{C}_{n} \\
C_{n}^{\gamma}
\end{array}\right]
$$

where $\hat{\gamma}_{n}$ is the standard matrix of anomalous dimensions and $\mathbf{K}_{n}=$ $\left(K_{n}^{N S}, K_{n}^{S}, K_{n}^{G}\right)$ leads to mixing of photonic and hadronic operators. One can find the explicit form of $\hat{\gamma}_{n}$ and $K$ up to terms $\alpha_{s} \ln Q^{2}$ in refs. $[12,13]$. (See also expressions 8-10 ). The term $\mathbf{K}_{n}$ gives an additional contribution in 
the Gribov-Lipatov-Altarelli-Parisi (GLAP) evolution equation to the quark distribution function from the direct quark and gluon production by photon (see Fig.3). \& Solving eq.(3) and substituting the result into (2) we can find the final expression for $F_{2}\left(Q^{2}\right)$. In symbolical notation it looks like (see, e.g. $[10,12])$ :

$$
\begin{aligned}
F_{2}^{n}\left(Q^{2}\right)= & \hat{C}_{n}\left(1, \bar{g}^{2}\right) \hat{M}^{n}\left(\bar{g}^{2}, \bar{g}_{0}^{2}\right)<\gamma\left|\hat{O}_{n}\left(Q_{0}^{2}\right)\right| \gamma>+ \\
& \hat{X}_{n}\left(\bar{g}^{2}, \bar{g}_{0}^{2}\right) \hat{C}_{n}\left(1, \bar{g}^{2}\right)+C_{n}^{\gamma}\left(1, \bar{g}^{2}\right)
\end{aligned}
$$

where

$$
\begin{gathered}
\bar{g}^{2}=4 \pi \alpha_{s}\left(Q^{2}\right) ; \quad \bar{g}_{0}^{2}=4 \pi \alpha_{s}\left(Q_{0}^{2}\right) ; \\
\hat{M}^{n}\left(\bar{g}^{2}, \bar{g}_{0}^{2}\right)=\exp \int_{\bar{g}}^{\bar{g}_{0}} d g \frac{\hat{\gamma}_{n}(g)}{\beta(g)} \\
\hat{X}=\int_{\bar{g}}^{\bar{g}_{0}} d g \frac{\mathbf{K}_{n}(g)}{\beta(g)} \cdot \hat{M}^{n}\left(\bar{g}^{2}, g^{2}\right)
\end{gathered}
$$

Functions

$$
\begin{gathered}
C_{n}^{S, N S}\left(1, \bar{g}^{2}\right)=\left(1+\frac{\bar{g}^{2}}{16 \pi^{2}} B_{n}^{\psi}\right) \cdot\left\{\begin{array}{l}
1 \text { for } N S \\
<e^{2}>\text { for } S
\end{array}\right. \\
C_{n}^{G}\left(1, \bar{g}^{2}\right)=<e^{2}>\frac{\bar{g}^{2}}{16 \pi^{2}} B_{n}^{G} \\
C_{n}^{\gamma}\left(1, \bar{g}^{2}\right)=\frac{\alpha}{4 \pi} 3 \sum_{i=1}^{f} e_{i}^{4} B_{n}^{\gamma} ; \quad<e^{2}>=(1 / f) \sum_{i=1}^{f} e_{i}^{2}
\end{gathered}
$$

$B_{n}^{\gamma}=(2 / f) B_{n}^{G}$ and $B_{n}^{G}$ are presented in [12].

In the general case $\hat{X}^{n}, \hat{M}^{n}, \hat{\gamma}_{n}, \mathbf{K}_{n}$ - are matrices. In what follows all calculations are made on an example of nonsinglet part of $F_{2}: F_{2}^{N S}$. In this case $M^{n}, \gamma_{n}, K_{n}$ are algebraic expressions. (The calculation of the singlet part is principally the same.)

For the nonsinglet case (4) is reduces to:

$$
F_{2}^{n(N S)}=C_{n}^{N S}\left(1, \bar{g}^{2}\right)\left\{M_{N S}^{n}\left(\bar{g}^{2}, \bar{g}_{0}^{2}\right)<\gamma\left|O_{n}^{N S}\left(Q_{0}^{2}\right)\right| \gamma>+X_{n}^{N S}\left(\bar{g}^{2}, \bar{g}_{0}^{2}\right)\right\}
$$

\footnotetext{
${ }^{1}$ It should be reminded that $\mathbf{K}$ play the role of anomalous dimensions and are $p^{2}$ independent. All $p^{2}$ dependence in (2) is gathered into matrix elements $\left\langle\gamma\left|\hat{O}_{n}^{i}\right| \gamma\right\rangle$ and appears in fact as a boundary condition.
} 
The factor in the curly brackets is usually treated as the moment of distribution function (see, however [21], when the other difinition of the distribution functions was discussed, in which $C_{n}^{N S}$ also was included in it). In the leading logarithmic approximation $C_{n}^{i}=1$ and the standard definition $F_{2}^{n}=\sum e_{i}^{2} q_{n}^{i}, \quad q_{n}^{i}=\int x^{n-1} q_{i}(x) d x$ is reproduced. In NLO the moments of quark distribution function $q^{N S}$ are defined by

$$
q_{n}^{N S}=\int x^{n-1} q^{N S}(x) d x=M_{N S}^{n}\left(\bar{g}^{2}, g_{0}^{2}\right)<\gamma\left|O_{n}^{N S}\right| \gamma>+X_{n}^{N S}\left(\bar{g}^{2}, g_{0}^{2}\right)
$$

In the same way the moments of singlet and gluon distribution functions are defined. The first term in (4) corresponds to the contribution from $C_{n}^{N S, S, G}\left(Q^{2} / \mu^{2}, g^{2}\right)$ in (2) (with account of the evolution (3)), the second and the third terms arise from $C_{n}^{\gamma}\left(Q^{2} / \mu^{2}, g\right)$ in (2) as a result of solution of eq.(3). Usually, the first term in (4) is treated as hadronic part of the structure function, since its form coincides with the solution of renormalization group equation for hadrons; the second term - as "point-like" production of quarks and gluons by photon (which involves characteristic $\ln Q^{2}$ behaviour) and the third term - is the proper photonic part which is included into quark distributions in some factorization schemes (e.g., $D I S_{\gamma}$, in ref.[16]). However, such separation may be dangereous because of the possibility of double counting, as was discussed in [1]. One can make this separation correctly basing on approach and results [1] where it is performed in the framework of QCD sum rules. To this end it is more convenient to use the evolution equation language.

The explicit form of the evolution equations for the distribution functions $q_{i}(x)$ and $G(x)$ was given in [14] in the leading logarithmic approximation while the next order terms were taken into account in $[11,15]$. These equations have the form: ( $i$ means flavours of quarks)

$$
\begin{gathered}
\frac{d q_{i}}{d t}=\frac{\alpha_{s}}{2 \pi}\left(P_{q g} \oplus q^{i}+P_{q q} \oplus G\right)+\frac{\alpha}{2 \pi} K_{q}^{i} \oplus \Gamma, \\
\frac{d G}{d t}=\frac{\alpha_{s}}{2 \pi}\left(P_{g q} \oplus \sum_{i}\left(q^{i}+\bar{q}^{i}\right)+P_{g g} \oplus G\right)+\frac{\alpha}{2 \pi} K^{G} \oplus \Gamma
\end{gathered}
$$

where $t=\ln Q^{2} / \Lambda^{2} ; P=P^{0}+\frac{\alpha_{s}\left(Q^{2}\right)}{4 \pi} P^{1}$, and $P$-are the splitting functions [11-13],

$$
K_{q}^{i}=\left(K_{q}^{i, 0}+\frac{\alpha_{s}}{2 \pi} K_{q}^{i, 1}\right) \quad K^{G}(x)=\frac{\alpha_{s}}{2 \pi} K_{G}^{1}(x)
$$


Here $P \oplus q$ stands for convolution

$$
\int_{x}^{1} \frac{d y}{y} P(x / y) q(y, t)
$$

$\Gamma(y)=\delta(1-y)$ is the photon distribution function in the photon. $\alpha / 2 \pi K_{q, G}(x)$ are related with anomalous dimensions $\mathbf{K}_{n}=\left(K_{n}^{N S}, K_{n}^{S}, K_{n}^{G}\right)$ by Mellin transformation. The exact relation can be found in paper [11]. We do not present it here since in what follows we shall need only $K_{q, G}(x)$, the explicit form of which is [11]:

$$
K_{q}^{i(0,1)}(x)=3 e_{i}^{2} \kappa_{q}^{(0,1)}(x), \quad i=u, d, s
$$

where $\kappa_{q}^{0}(x) \equiv \kappa(x)$ is defined in $(1)$;

$$
\begin{gathered}
\kappa_{q}^{1}(x)=\frac{2}{3} e_{i}^{2}\left\{4-9 x-(1-4 x) \ln x-(1-2 x) \ln ^{2} x+4 \ln (1-x)+\quad(9)\right. \\
\left.+\left[4 \ln x-4 \ln x \ln (1-x)+2 \ln ^{2} x-4 \ln (1-x)+2 \ln ^{2}(1-x)-(2 / 3) \pi^{2}+10\right] \kappa(x)\right\}
\end{gathered}
$$
$K_{G}^{1}(x)$ was taken from [16] where the error in [12] and in the papers based on it was corrected

$$
K_{G}^{1}(x)=\sum_{i} e_{i}^{2} \cdot 4\left[-16+8 x+\frac{20}{3} x^{2}+\frac{4}{3 x}-(6+10 x) \ln x-2(1+x) \ln ^{2} x\right]
$$

Here, analogously to (6), the function $F_{2}$ is determined through quark and gluonic functions as $[15,11]$

$$
\begin{gathered}
F_{2}(x)=x\left\{\sum_{i} e_{i}^{2}\left(\hat{q}_{i}(x)+\frac{\alpha_{s}}{4 \pi} B_{\psi} \oplus \hat{q}_{i}\right)+\right. \\
\left.+<e^{2}>\cdot \frac{\alpha_{s}}{4 \pi} B_{G} \oplus G+3 \sum_{i} e_{i}^{4} B_{\gamma}(x) \alpha / 4 \pi\right\}
\end{gathered}
$$

where $\hat{q}=q+\bar{q}$

$$
\begin{gathered}
B_{\psi}(x)=(8 / 3)\left\{\frac{9+5 x}{4}-\frac{1+x^{2}}{1-x} \ln x-\frac{3}{4}\left[\frac{1+x^{2}}{1-x}\right]_{+}+\right. \\
\left.+\left(1+x^{2}\right)\left[\frac{\ln (1-x)}{1-x}\right]_{+}-\left(9 / 2+\pi^{2} / 3\right) \delta(1-x)\right\}
\end{gathered}
$$




$$
\begin{gathered}
B_{G}(x)=2 f\left(\left(1-2 x+2 x^{2}\right) \ln \frac{1-x}{x}-1+8 x(1-x)\right) ; \\
\left.B_{\gamma}(x)=(2 / f) \cdot B^{G}(x)\right)
\end{gathered}
$$

$B_{n}^{\psi}$ and $B_{n}^{\gamma}$ defined in (5) are $n$ - moments of these functions. (All expressions here are in the $\overline{M S}$ scheme). The last terms in eqs.(7) correspond to the process of point-like (direct) production of quarks and gluons from photon in LO and NLO: we preserve only the terms proportional to $\ln Q^{2}$ or $\alpha_{s} \ln Q^{2}$ (see Fig.3).

In the case of $D I S_{\gamma}$ scheme suggested in Ref.[16]

$$
\begin{aligned}
& F_{2}^{D I S_{\gamma}}(x)=x\left\{\sum_{i} e_{i}^{2}\left(\hat{q}^{D I S_{\gamma}}(x)+\frac{\alpha_{s}}{4 \pi} B_{\psi} \oplus \hat{q}^{D I S_{\gamma}}(x)\right)+\right. \\
& \left.+<e^{2}>\frac{\alpha_{s}}{4 \pi} B_{G} \oplus G(x)\right\}
\end{aligned}
$$

where the last term in (11) is included in the $\hat{q}^{D I S_{\gamma}}(x)$. This redefinition leads to transformation of $K_{q, G}^{(1)}\left(\equiv K_{q}^{i, 1}\right.$ or $K_{G}^{1}$, see $\left.8-10\right)$.

$$
K_{q, G}^{(1) D I S_{\gamma}}=K_{q, G}^{(1)}+\delta K_{q, G}
$$

where $\delta K_{q, G}$ from eq.(3.1, A.11-A.12) in ref.[16]. This scheme has many advantages in comparison with the $\overline{M S}$ scheme (see discussion in [16]).

Especially in the $D I S_{\gamma}$ scheme there is no strong difference between LO and NLO results. Hereafter we will work with the $D I S_{\gamma}$ scheme (and omit $D I S_{\gamma}$ indeces). We would like to stress, however, that our results are comparatively insensitive to the choice of renormalization scheme $\left(\overline{M S}\right.$ or $\left.D I S_{\gamma}\right)$, see discussion at the end of the paper.

The evolution equations (7) are equivalent to the renormalization group equations for the moments (3). The form of equations (3) and (7) is independent of the photon virtuality, the $p^{2}$ dependence is hidden in the matrix elements $<\gamma\left|\hat{O}_{n}\right| \gamma>$ and therefore appears only through the boundary conditions. Evidently, for virtual photon the renormalization group equations are valid at $Q^{2}>>-p^{2}$. In what follows we consider only the case of transversally polarized real or virtual photon and for simplicity the notation $F_{2}$ will be used for $F_{2}^{T}$ at $p^{2} \neq 0$. The unpolarized virtual photon case will be discussed at the end of Section 3 .

The evolution equations (7) are inhomogeneous. As is known, the general solution of inhomogeneous equation is given by the sum of partial solution of 
inhomogeneous equation plus general solution of homogeneous equation. The latter coincides with the standard solution of evolution equation for hadrons. We choose the partial solution of evolution equation in NLO by requiring its vanishing at $Q^{2}=Q_{0}^{2}$ (see refs.[15-17]). The solution of inhomogeneous equations, represented in terms of moments of singlet and gluon distributions is given by [16]

$$
\begin{gathered}
{\left[\begin{array}{c}
q_{n}^{s} \\
G_{n}
\end{array}\right]=\frac{\alpha}{2 \pi} \frac{2}{\beta_{0}}\left\{\left[2 \pi / \alpha_{s} \hat{P}_{+}-\frac{2}{\beta_{0}} \hat{P}_{+} \hat{P}_{1} \hat{P}_{+}+\right.\right.} \\
\left.+\frac{\beta_{1}}{\beta_{0}^{2}} \lambda_{+} \hat{P}_{+}+\frac{\hat{P}_{-} \hat{P}_{1} \hat{P}_{+}}{\lambda_{+}-\lambda_{-}-\beta_{0} / 2}\right] \times \\
\times \frac{1-L^{1-2 \lambda_{+} / \beta_{0}}}{1-2 \lambda_{+} / \beta_{0}} \hat{K}_{0}-\frac{1-L^{-2 \lambda_{+} / \beta_{0}}}{2 \lambda_{+} / \beta_{0}} \cdot\left[\hat{P}_{+} \hat{K}_{1}-\frac{\beta_{1}}{2 \beta_{0}} \hat{P}_{+} \hat{K}_{0}-\right. \\
\left.+\frac{2}{\beta_{0}}\left(\hat{P}_{+} \hat{P}_{1} \hat{P}_{+}-\frac{\beta_{1}}{2 \beta_{0}} \lambda_{+} \hat{P}_{+}-\frac{\beta_{0}}{2} \frac{\hat{P}_{+} \hat{P}_{1} \hat{P}_{-}}{\lambda_{-}-\lambda_{+}-\beta_{0} / 2}\right) \hat{K}_{0}\right]+ \\
\left.+\left(\text { terms with interchange } \lambda_{+} \rightarrow \lambda_{-}, \hat{P}_{+} \rightarrow \hat{P}_{-}\right)\right\}
\end{gathered}
$$

Here $\hat{P}_{1}, \hat{P}_{+}, \hat{P}_{-}$are matrices; $q_{n}^{s}=\sum_{i=1}^{f} q_{n}^{i}+\bar{q}_{n}^{i}$

$$
\begin{gathered}
\hat{P}_{1}=\left(\begin{array}{cc}
P_{q q}^{1}, & P_{q g}^{1} \\
P_{g q}^{1} & P_{g g}^{1}
\end{array}\right), \quad \hat{P}_{ \pm}= \pm \frac{\hat{P}_{0}-\lambda_{\mp} \cdot I}{\lambda_{+}-\lambda_{-}}, \quad \hat{P}_{0}=\left(\begin{array}{cc}
P_{q q}^{0} & \tilde{P}_{q g}^{0} \\
P_{g q}^{0} & P_{g g}^{0}
\end{array}\right) \\
\lambda_{ \pm}=1 / 2\left(P_{q q}^{0}+P_{g g}^{0} \pm \sqrt{\left.\left(P_{q q}^{0}-P_{g g}^{0}\right)^{2}+4 \tilde{P}_{q g}^{0} P_{g q}^{0}\right)}, \quad \tilde{P}_{q g}^{0}=2 f P_{q g}^{0},\right. \\
\hat{K}_{0}=\left(\begin{array}{c}
K_{q}^{0} \\
0
\end{array}\right), \quad \hat{K}_{1}=\left(\begin{array}{c}
K_{q}^{1} \\
K_{G}^{1}
\end{array}\right), L=\frac{\alpha_{s}\left(Q^{2}\right)}{\alpha_{s}\left(Q_{0}^{2}\right)} ; \quad K_{q}^{(0,1)}=\sum_{1=1}^{2 f} K_{q}^{i(0,1)}
\end{gathered}
$$

$P_{q q}^{0,1}, P_{q g}^{0,1}$ etc. mean the n-th moment of the standard splitting functions.

As mentioned in refs.[15,16], such a choice of the partial solution when even at large $Q^{2}$ the terms $L^{-2 \lambda_{ \pm} / \beta}$ are retained permits one to get rid of nonphysical poles at $n=1.596$ and $n=2$ corresponding to $1-2 \lambda_{+} / \beta_{0}=0$ and $\lambda_{+}=0$. These poles would correspond to the poles in $\mathrm{x}$-distribution of the type $1 / x^{1.596}$ and $1 / x^{2}$. The vanishing at the same points numerators result in the logarithmic divergences in $\mathrm{x}$-distributions instead of power-like ones. The partial solution chosen in this way is completely analogous to 
the term $\hat{X}\left(\bar{g}^{2}, g_{0}^{2}\right)$ in the solution of eq.3. As already has been mentioned, this term incorporates the terms proportional to $\ln Q^{2}$, related to the pointlike mechanism of quark and gluon production by photon in LO and NLO. However, it would be erroneous to think that the solution of inhomogeneous equation with zero boundary conditions takes into account the whole contribution from the process of the point-like quark production at a given $Q^{2}$ while the general solution of homogeneous equation (equivalent of $M^{n}$ in (4)) - only hadronic part, i.e. the contribution of soft and collinear quarks . . At such a choice, there may appear a double counting (or a deficit if we take hadronic part to be zero [16]).

One of the methods to solve this problem correctly was discussed in the Introduction. This is to use as boundary conditions the results of ref. [1] eq.(1),, where the point-like and hadronic contributions into photon structure functions - respectively, the first and the second terms in curly brackets in (1) - are calculated in QCD. In such case the mechanism of the pointlike production corresponds to solution of inhomogeneous equation with the boundary conditions from the first term of (1) to which one should add the hadronic part corresponding to solution of homogeneous equation with the boundary conditions from the second term of (1).

Owing to linearity of the evolution equations, it is, naturally, more convenient to take the solution of inhomogeneous equation with the zero boundary conditions and to add the solution of homogeneous equation with the boundary conditions which are the sum of the boundary conditions of the point-like and hadronic parts. But in doing so, the solution of homogeneous equation is not only the contribution of hadronic part (e.g., it is, erroneous to choose in this case the boundary condition from VDM considerations).

Before going to solution of homogeneous equation, let us come back once more to the general form of inhomogeneous equation (7).

Consider two functions $\varphi(x) \equiv q^{N S}(x)=(1 / 2)(u(x)-d(x))$ and $\tau=$ $-(1 / 2)\left(u / e_{u}^{2}-d / e_{d}^{2}\right)$ where $e_{i}$ is the charge square of the corresponding quark. The evolution equation (7) and the boundary condition on $F_{2}^{T}$ are the same for $d$ and $s$ quarks besides small difference in the boundary condition (1) arising from the mass difference of $\rho$ and $\varphi$ mesons. (The calculation shows that the influence of this difference on gluon distribution is very small)

Thus, we may safely take $d(x)=s(x)$, and, correspondingly,

\footnotetext{
${ }^{2}$ Such a distinction may be achieved, as we shall see in what follows, but only at certain boundary conditions.
} 


$$
d(x)=s(x), \quad \hat{q}_{i} \equiv\left(q_{i}+\bar{q}_{i}\right)=4 \varphi \frac{e_{i}^{2}}{\Delta}+\frac{4 e_{u}^{2} e_{d}^{2}}{\Delta}
$$

where $\Delta=e_{u}^{2}-e_{d}^{2}=1 / 3$. The inhomogeneous evolution equation (7) expressed through these functions is:

$$
\begin{gathered}
\frac{d \varphi(x, t)}{d t}=\frac{\alpha}{2 \pi} \frac{1}{2}\left(e_{u}^{2}-e_{d}^{2}\right) 3\left(k_{q}^{0}(x)+\frac{\alpha_{s}(t)}{2 \pi} k_{q}^{(1)}(x)\right)+ \\
+\frac{\alpha_{s}(t)}{2 \pi} \int_{x}^{1} \frac{d y}{y} P_{q q}(x / y) \varphi(y, t) \\
\frac{d \tau(x, t)}{d t}=\frac{\alpha_{s}(t)}{2 \pi} \int_{x}^{1}\left(P_{q q}(x / y) \tau(y, t)+P_{q g}(x / y) \frac{1}{2} \frac{\Delta}{e_{u}^{2} e_{d}^{2}} G(y, t)\right) \frac{d y}{y} \\
\frac{d G(x, t)}{d t}=\frac{\alpha \alpha_{s}(t)}{(2 \pi)^{2}} K_{G}^{1}(x)+\frac{\alpha_{s}(t)}{2 \pi} \int_{x}^{1}\left[P _ { q G } ( x / y ) \left(\frac{4 \Sigma e_{i}^{2}}{\Delta} \varphi(y, t)+\right.\right. \\
\left.\left.+\frac{4 f e_{u}^{2} e_{d}^{2}}{\Delta} \tau(y, t)\right)+P_{g g}(x / y) G(y, t)\right] \frac{d y}{y}
\end{gathered}
$$

where notations are the same as in (7), (7a), (8). The evolution equation for $\tau(x)$ has the form of standard evolutions for hadrons and all quark and gluon point-like production is now absorbed in $\varphi$ function. Thus, $\varphi(x)$ may be treated as valence quark distribution in the photon and $4\left(e_{u}^{2} e_{d}^{2} / \Delta\right) \tau(x)$ as "sea quark" distribution [?. In the next Section we consider two variants of boundary conditions and discuss the obtained solutions.

3. Solution of Evolution Equations. Discussion of Results.

In the first variant of the boundary conditions we assume that at some, relatively small $Q^{2}=Q_{0}^{2}$ there are no $\tau$ ("sea") quarks, as well as gluons but there are only "valence" quarks ( $\varphi$-quarks).

Physically, this corresponds to assumption that both gluons and sea quarks in photon are produced in perturbative way due to their emission by valence quark (as well by a bare photon for the gluon case) and they are absent at the low normalization point $Q^{2}=Q_{0}^{2} \sim 1 G e V^{2}$. As has been already noticed in the Introduction, an analogous idea was forwarded to describe the structure function of hadrons where it was supposed [9], that at small $Q^{2}=Q_{0}^{2} \sim 1 \mathrm{GeV}^{2}$ a hadron consists only of valence quarks. But such an

\footnotetext{
${ }^{3}$ Such detemination of "valence" and "sea" quarks distribution is close to that used in [20].
} 
idea failed in description of the hadron structure functions - it appears to be inconsistent with experiment. This idea has better chances for photon since it can be naturally thought that photon has no gluons and sea quarks (in the sense defined at the end of the previous Section) as their constituents. Note that for virtual photon $Q^{2}$ should be chosen larger than $\left|p^{2}\right|$. As the hadronic component contribution into structure function (1) decreases rapidly with $\left|p^{2}\right|$ increase, and hence it follows that for strongly virtual photon our requirement is fulfilled automatically.

The total solution for the quark distribution function consists of the sum of solution of inhomogeneous equation which was under discussion in the previous section and of the solution of homogeneous equation

$$
\begin{gathered}
\frac{d \varphi(x, t)}{d t}=\frac{\alpha_{s}(t)}{2 \pi} \int_{x}^{1}\left(P_{q q}(x / y) \varphi(x, t)\right) \frac{d y}{y} \\
\frac{d G(x, t)}{d t}=\frac{\alpha_{s}(t)}{2 \pi} \int_{x}^{1}\left\{\left[P _ { q G } ( x / y ) \left(\frac{4 \Sigma e_{i}^{2}}{\Delta} \varphi(y, t)+\right.\right.\right. \\
\left.\left.\left.+\frac{4 f e_{u}^{2} e_{d}^{2}}{\Delta} \tau(y, t)\right)\right]+P_{g g}(x / y) G(y, t)\right\} \\
\frac{d \tau(x, t)}{d t}=\frac{\alpha_{s}(t)}{2 \pi} \int_{x}^{1}\left(P_{q q}(x / y) \tau(y, t)+\frac{1}{2} P_{q q}(x / y) \frac{\Delta}{e_{u}^{2} e_{d}^{2}} G(y, t)\right) \frac{d y}{y}
\end{gathered}
$$

with the boundary conditions from (1). Since we restrict ourselves to NLO of inhomogeneous equation i.e., to terms $\alpha_{s} \ln Q^{2}$, then in the same order the homogeneous equation can be solved in the leading logarithmic approximation, so $\frac{\alpha_{s}(t)}{2 \pi}=\frac{2}{\beta_{0} t}$. In this approximation in eq.(11a) for $F_{2}\left(x, Q_{0}^{2}\right)$ (note, that $G\left(x, Q_{0}^{2}\right)=0$ )

$$
(1 / x) F_{2}\left(x, Q_{0}^{2}\right)=\sum e_{i}^{2}\left[\hat{q}_{i}(x)+\frac{\alpha_{s}}{4 \pi} B_{\psi} \oplus \hat{q}_{i}(x)\right]
$$

one should omit the term $\left(\alpha_{s} / 4 \pi\right) B_{\psi} \oplus \hat{q}_{i}$, since the solution of homogeneous equation $\hat{q}_{i}\left(x, Q^{2}\right)$ has no $\ln Q^{2}$ and the contribution from the solution of inhomogeneus equation at $Q^{2}=Q_{0}^{2}$ is zero. As the estimates show, this term gives a small contribution to the gluonic structure function at $x<0.5$. Therefore the boundary conditions have the form

$$
\begin{gathered}
F_{2}\left(x, Q_{0}^{2}\right)=x \sum e_{i}^{2} q_{i}\left(x, Q_{0}^{2}\right) \\
G\left(x, Q_{0}^{2}\right)=\tau\left(x, Q_{0}^{2}\right)=0
\end{gathered}
$$


Accounting for (1), (13) we have

$$
\begin{gathered}
\varphi\left(x, t_{0}\right)=\frac{\alpha}{4 \pi}\left\{\left[2+\kappa(x)\left(\ln \frac{Q_{0}^{2}}{x^{2}\left(s_{0}-p^{2}\right)}-3+\frac{p^{2}}{s_{0}-p^{2}}\right)\right]+\right. \\
\left.+\frac{s_{0}^{2}}{2\left(m_{V}^{2}-p^{2}\right)^{2}} \rho(x)\right\}
\end{gathered}
$$

where $t_{0}=\ln \left(Q_{0}^{2} / \Lambda^{2}\right)$

$$
\rho(x)=\kappa(x)-\frac{8 \pi^{2}}{27 x^{2} s_{0}^{2}}<0\left|\frac{\alpha_{s}}{\pi} G_{\mu \nu}^{2}\right| 0>
$$

and $m_{V}$ means the weighted average of $\rho$ and $\varphi$ mass. Let us represent $\varphi(x, t)$ as a series

$$
\varphi(x, t)=\varphi\left(x, t_{0}\right)+\sum_{1}^{\infty} \frac{\varphi_{m}(x)}{m !}\left(\ln \frac{t}{t_{0}}\right)^{m}
$$

For $\tau(x, t)$ and $G(x, t)$ similar expressions take place:

$$
\tau(x, t)=\sum_{1}^{\infty} \frac{\tau_{m}(x)}{m !}\left(\ln \frac{t}{t_{0}}\right)^{m}, \quad G(x, t)=\sum_{1}^{\infty} \frac{G_{m}(x)}{m !}\left(\ln \frac{t}{t_{0}}\right)^{m}
$$

Substituting (19),(20) into the evolution equations we get recurrent relations $(f=3)$

$$
\begin{gathered}
\varphi_{m}(x)=\left(\frac{2}{\beta_{0}}\right)^{m} \underbrace{P_{q q}^{0} \oplus P_{q q}^{0} \oplus \ldots P_{q q}^{0}}_{m \text { times }} \oplus \varphi\left(x, t_{0}\right) \\
G_{m+1}(x)=\frac{2}{\beta_{0}}\left[\frac{4}{\Delta} \sum e_{i}^{2} P_{g q}^{0} \varphi_{m}(x)+\frac{12 e_{u}^{2} e_{d}^{2}}{\Delta} \tau_{m}(x) \oplus P_{g q}^{0}+P_{g g}^{0} \oplus G_{m}(x)\right] \\
\tau_{1}(x)=0 \\
\tau_{m+1}(x)=\frac{2}{\beta_{0}}\left[P_{q q}^{0} \oplus \tau_{m}(x)+\frac{1}{2} \frac{\Delta}{e_{u}^{2} e_{d}^{2}} P_{q g} \oplus G_{m}(x)\right]
\end{gathered}
$$

In order to solve equations (21) it is necessary to know the function $\varphi\left(x, Q_{0}^{2}\right) \equiv$ $\varphi\left(x, t_{0}\right)$ in the region $0.75<x<1$ where eqs.(1) and (18) are invalid. Notice that the uncertainty at large $x$ arises only in the second term in (1) or (18) related to the vector meson contribution. The natural extrapolation comes from quark counting rules and corresponds to $f_{\rho}(x) \sim(1-x)$ at large $x$. We considered also the other forms of extrapolation $f_{\rho}$ in the domain of large 
$x: f_{\rho}(x)=A(1-x)^{\alpha}$, where $A$ was determined from the matching with the second term in (1) at the point $x=0.75$. It was found that $G\left(x, Q^{2}\right)$ at $x \leq 0.5$ varies only by a few per cent if $\alpha$ varied from 1 to 2 . We emphasize, that the account in the evolution equations of singular terms, proportional to $\delta(1-x), \delta^{\prime}(1-x)$ etc, (see [1]) would be a mistake. These terms (e.g. the term, proportional to operator $\alpha_{s} \bar{\psi} \Gamma_{i} \psi \bar{\psi} \Gamma_{i} \psi$ ) formally appear in the OPE over the soft photon virtuality $1 / p^{2}$. However, as was explained in ref.3, these terms are unphysical because they correspond to the bump at $x=1$ and must be compensated in the correct theory.

The series (19),(20) rapidly converge at $x \leq 0.6$ and $Q^{2}<100 \mathrm{GeV}^{2}$, so it is sufficient to take into account three terms in the expansion. In the homogeneous equation we can put the one-loop expression for $\alpha_{s}$ : $\alpha_{s}=$ $4 \pi / \beta_{0} \ln \left(Q^{2} / \Lambda^{2}\right), \Lambda=230 \mathrm{MeV}$, since this equation is treated in the leading logarithmic approximation. In the case of inhomogeneous equation (10) the calculations are performed in NLO in $D I S_{\gamma}$ regularization scheme and, correspondingly, two-loop expression for $\alpha_{s}$ is used:

$$
\frac{1}{4 \pi} \alpha_{s}\left(Q^{2}\right)=\frac{1}{\beta_{0} \ln \left(Q^{2} / \Lambda^{2}\right)}-\frac{\beta_{1}}{\beta_{0}^{3}} \frac{\ln \ln \left(Q^{2} / \Lambda^{2}\right)}{\ln ^{2}\left(Q^{2} / \Lambda^{2}\right)}
$$

The functions $q(x), G(x)\left(q(x) \equiv q^{s}(x)\right.$ is the singlet quark distribution) were obtained from the solution of the inhomogeneous equation (12) by the inverse Mellin transformation. The results are presented in Fig.4-8. Dotted lines correspond to gluon condensate contribution, thin solid lines - to hadronic part and thick lines - total gluon distribution $x G(x) / \alpha$. For $p^{2} \neq 0($ Fig. $5 \div 7)$ thick solid lines correspond to transversally polarized photon case, and dashed lines - unpolarized photon case, which will be discussed at the end of the paper.

As is seen from Figs.4-7 the negative gluonic condensate contribution is essential only at $x<0.1$, it increases steeply with $x$ decreasing and for the real photon at $Q^{2}=5 G e V^{2}$ comprises a half of the total $G(x)$ at $x \approx 0.02$. This means that the QCD sum rule approach prediction for the gluon distribution function in the real photon is reliable up to $x>0.02-0.05$ (in the framework of the chosen boundary condition - variant $\mathrm{I}(\mathrm{HI})$ ). The gluonic condensate contribution decreases rapidly with increasing of photon virtuality $-p^{2}$.

As was discussed in the Introduction, such an essential extension of the applicability region towards small $x$ is possible for the gluon distribution function only. For quark distribution the applicability region starts at larger 
$x>0.1$.

As is seen from Figs.4-7, the hadronic part is rather large for the real photon $\left(\sim 50 \%\right.$ at $Q^{2}=5 \mathrm{GeV}^{2}$ and $30 \%$ at $\left.Q^{2}=50 \mathrm{GeV}^{2}\right)$ and decreases rapidly with $\left|p^{2}\right|$ increasing.

An approach similar to ours for the case of the virtual photon was developed in refs.[15,17]. Here, however, the hadronic part was not singled out from the boundary conditions and considered separately, as we did.

The dashed lines in Fig. 4 at $Q^{2}=5$ and $50 \mathrm{GeV}^{2}$ represent the results of ref.[16], where the NLO evolution equations were solved with the zero boundary conditions for inhomogeneous as well as for homogeneous equations. As is expected, they are much more less than our results although relative difference decreases with $x$ increasing. The difference is large even at $Q^{2}=50 \mathrm{GeV}^{2}$ what means that the domain of $Q^{2} \sim 50 \mathrm{GeV}^{2}$ is far from asymptotics, where dominant are the terms in the solution of inhomogeneous equations $\left(\sim \ln Q^{2}\right.$ and $\alpha_{s} \ln Q^{2}$ ) independent of the boundary conditions.

Our results for the gluon distribution function are weakly dependent on the choice of the normalization point $Q_{0}^{2}$. For the real photon this can be seen from Fig.8. With $Q^{2}$ or $\left|p^{2}\right|$ increasing the $Q^{2}$ dependence becomes even weaker. The LO results are not too much different from NLO: the difference is less than 10-15\% almost everywhere except for the highest considered virtuality $\left|p^{2}\right|=2 G e V^{2}$ where it is about $30 \%-40 \%$. For this reason the LO results are not shown in the Figures.

We turn now to the variant $\mathrm{II}(\mathrm{H} 2)$ of the boundary conditions discussed in the Introduction. The second term in the photon structure function, eq.1, corresponds to the target photon into vector meson transition with subsequent projectile virtual photon-vector meson scattering. At such an interpretation it is natural to assume that at low $Q^{2}$ there are nonperturbative gluons in the photon and their distribution is determined by gluon distributions in vector mesons. As was shown in ref.[1], the second term in (1) is related to the transverse structure function $f_{V}^{T}(x)$ of the fictitious vector meson $V$ built from one flavour quark $q$ with unit charge

$$
\frac{4 \pi}{g_{V}^{2}} f_{V}^{T}(x) / x=\frac{3}{2 \pi} \frac{s_{0}^{2}}{m_{V}^{4}} \rho(x)
$$

where $\rho(x)$ is given by (18) and $g_{V}^{2}$ is the $\gamma-V$ transition coupling constant. (Experimentally, $g_{V}^{2} / 4 \pi=1.27$.) $f_{V}^{T}(x) / x$ is equal to quark distribution, 


$$
f_{V}^{T}(x)=x[q(x)+\bar{q}(x)]
$$

In a similar way we can define the gluon distribution $G_{V}^{T}(x)$ in $V$-meson. Assuming the standard form of $x$-dependence, $x G_{V}^{T}(x) \sim(1-x)^{3}$, we have

$$
x G_{V}^{T}(x)=c \frac{3}{8 \pi^{2}} \frac{s_{0}^{2}}{m_{V}^{4}} g_{V}^{2}(1-x)^{3}
$$

where the normalization constant $c$ is determined from the requirement that gluons in $V$-meson carry $40 \%$ of $V$-meson momentum, $c=0.42$. The gluon distribution in the virtual photon is related to $x G_{V}^{T}(x)$ by

$$
\begin{gathered}
x G_{\gamma}^{T}\left(x, Q_{0}^{2}\right)=\frac{4 \pi \alpha}{g_{V}^{2}} \Sigma e_{q}^{2} \frac{m_{V}^{4}}{\left(m_{V}^{2}-p^{2}\right)} x G_{V}^{T}(x)= \\
=c \frac{3 \alpha}{2 \pi} \Sigma e_{q}^{2} \frac{s_{0}^{2}}{\left(m_{V}^{2}-p^{2}\right)^{2}}(1-x)^{3}
\end{gathered}
$$

Eq.24 is used as the boundary condition for the gluon distribution in the variant II of boundary conditions. In the same way the boundary conditions for sea quark distributions can be also imposed. But since they carry only $10 \%$ of the vector meson momentum, their contribution to the final gluon distribution is negligibly small. We emphasize that (24) is the boundary condition for the hadronic part of the gluon distribution function, for nonhadronic, point-like part the boundary condition is still zero. As before, the boundary conditions (17) are used for valence quark distributions. (The account of the order $\alpha_{s}$ gluonic contribution to $F_{2}\left(x, Q_{0}^{2}\right)$ in (17) is beyond the accuracy of our calculation and, as the estimate shows, this contribution is small numerically).

The results of the calculations in the variant II are presented in Figs.9-12. All notations are the same, as in Fig. $4 \div 8$. In this variant the hadronic part dominates in the gluonic contribution function for the real or virtual photon even at $Q^{2}=50 G e V^{2}$. In comparison with the variant I $G\left(x, Q^{2}\right)$ is essentially larger, especially in the case of the real photon - by a factor of 2-3. The relative difference of $G\left(x, Q^{2}\right)$ in variants I and II decreases with $Q^{2}$ increased as a result of increasing role of point-like gluon production. The difference in gluon distributions in variants I and II is going down steeply with increasing of the photon virtuality $\left|p^{2}\right|$.

The results of ref.[18] at $Q^{2}=10 \mathrm{GeV}^{2}$ are shown in Fig.13 by dotted line. The method of the calculations of ref.[18] is different from ours in three 
aspects: i)low normalization point in inhomogeneous differential equation with the zero boundary condition at $Q^{2}=0.3 \mathrm{GeV}^{2}$ in [18] comparing with our $Q_{0}^{2}=1 \mathrm{GeV}^{2}$; ii) our boundary gluonic distribution (24) is much more concentrated at small $x$ than that used in ref.[18] $-x G_{\gamma}(x) \sim x^{0.5}(1-x)^{\alpha}$, where $\alpha=0.1$ at normalization point $Q_{0}^{2}=0.3 \mathrm{GeV}^{2}$ and $\alpha \sim 1$ at $Q^{2} \sim$ $5 \div 10 \mathrm{GeV}^{2}$; iii) the difference in the boundary conditions for valence quarks - eq.(17).

The change of the normalization point to $Q_{0}^{2}=2 G e V^{2}$ (instead of $Q_{0}^{2}=$ $1 \mathrm{GeV}^{2}$ ) decrease gluonic distribution in our calculation by $10-12 \%$ (Fig.8b). Therefore, the $Q_{0}^{2}$ dependence of the final gluon distribution is weaker in the variant II than in the variant $I$.

The results of ref.[22] are shown by dashed line in Fig.13, and results of [23] -by crosses.

The negative gluon condensate contribution to the boundary condition for valence quarks (eq.17) results in flattering of $G(x)$ at small $x \sim 0.02-0.05$. Result of fit, made in [20], are higher than ours (in all six choises of their input) at low $x$, because their boundary condition for $G(x)$ is $2.5 \div 5$ times larger, than ours. In Fig.14 we compare our results (II variant, H2) whith three sets of boundary conditions, offered by [20]. (Three other sets are even more larger and we don't show them).

In the considered above two variants of the boundary conditions $(17,18)$ and $(18,24)$ the gluonic distributions in the real or weakly virtual photon $\left(\left|p^{2}\right| \leq 0.5 \mathrm{GeV}^{2}\right)$ are essentially different. It is impossible to remove this difference by varying the normalization point $Q_{0}^{2}$ in the first variant of the boundary conditions. The experimental investigation of the gluon distribution in photon can shed light on the problem if there are or not intrinsic gluons in the photon.

The calculation of the whole $F_{2}$ structure function can be done in a similar way. Like the gluon distribution, quark distribution functions are given by the sum of partial solutions of inhomogeneous equations with boundary conditions $q^{i n h}\left(x, Q_{0}^{2}\right)=0$ plus general solution of homogeneous equation $q^{\text {hom }}\left(x, Q^{2}\right)$ with boundary conditions, following from (1). As we are interested only in the terms, proportional to $\ln Q$ or $\alpha_{s} \ln Q$, so the gluon contribution and $\alpha_{s}$-corrections to quarks contributions in (11a) should be calculated in $\ln Q^{2}$ order. This means, that this contributions may be accounted only in the solution of inhomogeneous equations since the terms, proportional to $\ln Q^{2}$, arises only there. So, if we write $F_{2}(x)$ in the form 


$$
F_{2}\left(x, Q^{2}\right)=F_{2}^{i n h}\left(x, Q^{2}\right)+F_{2}^{h o m}\left(x, Q^{2}\right)
$$

where

$$
\begin{aligned}
F_{2}^{i n h}\left(x, Q^{2}\right)= & x\left\{\sum _ { i } e _ { i } ^ { 2 } \left(\hat{q}_{i}^{i n h}\left(x, Q^{2}\right)+\frac{\alpha_{s}}{4 \pi} B_{\psi} \oplus \hat{q}_{i}^{i n h}\left(x, Q^{2}\right)+\right.\right. \\
& \left.+<e^{2}>\frac{\alpha_{s}}{4 \pi} B_{G} \oplus G\left(x, Q^{2}\right)\right\}
\end{aligned}
$$

then

$$
F_{2}^{\text {hom }}\left(x, Q_{0}^{2}\right)=x \sum_{i=1}^{f} e_{i}^{2} \hat{q}_{i}^{\text {hom }}\left(x, Q_{0}^{2}\right)
$$

and the boundary conditions for $\hat{q}^{\text {hom }}\left(x, Q_{0}^{2}\right)$ are the same as $(17)$ for the I variant(the II variant gives almost the same results and we will not discuss it).

The results for $F_{2}$ are shown in Figs.15-18. (The notations are the same as for the gluon distribution function, Figs.4-7). The results for the real photon case (thick solid line in Figs.15,16) are compared with the experimental data of TPC/2 $\gamma[24,25]$, PLUTO [26-28] and OPAL [29] collaborations. For comparison the results of [18] (dashed line) and [20] (crossed line) are shown. One can see, that our results are in a good agreement with the data at $0.2<x<0.7$ and $Q^{2}=5 \div 41 G e V^{2}$ where our approach is valid. At $x<0.2$ the gluon condensate contribution becomes too large and our results are unreliable. We should notice that, of course, at $Q^{2} \geq 40 \div 50 \mathrm{GeV}^{2}$ the role of $c$-quarks is not negligible at $x<0.3$ and one has to take them into consideration.

The results of the structure function $F_{2}$ calculations for virtual photon are presented in Figs.17,18. The thick solid line corresponds to the transversally polarized photon case, the dashed one - to the unpolarized virtual photon case (the latter will be discussed below).

Note that our results comparatively weekly depend on the renormalization scheme $\left(M \bar{S}\right.$ or $\left.D I S_{\gamma}\right)$, as it can be seen from Fig.19, where our results are plotted in these two schemes. An approximate scheme independence arises because the scheme difference in the solution of inhomogeneous equation (see [16]) is compensted by the opposite difference of the solution of homogeneous equation. The main reason for this circumstance is that we have no separate boundry conditions for homogeneous equation (which is independent of the 
solution of inhomogeneous equation and is determined from any physical ideas like VDM), but have boundary condition for total $F_{2}\left(x, Q_{0}^{2}\right)$.

Finally, let us discuss the nonpolarized virtual photon case. It is reduced to trivial redefinition of boundary conditions for homogeneous equation $(17,18)$. Here we now should use $F_{2}=F_{2}^{T}+(1 / 2) F^{L}, \quad F_{2}^{T}$ being defined in (1) and $F_{2}^{L}$ can be found in [1]

$$
F_{2}^{L}=\frac{3 \alpha}{\pi} \sum e_{i}^{4} 4 x^{2}(1-x) \frac{p^{2}}{p^{2}-\frac{m_{q}^{2}}{x(1-x)}}
$$

$m_{q}$ - is the quark mass.

Note that there is no contribution from gluon condensate in $F_{2}^{L}$ (see [1]).

The results for the unpolarized virtual photon case are about $20 \%$ higher than for transversally polarized one and are shown by dashed line in Figs.5-7, 10-12 for gluon distribution and in Figs.17,18 for $F_{2}$ structure functions. For comparison, the results of [19] (disregarding any heavy quark contribution) are shown in Figs.10,11,18 by crossed line.

One of the authors (A.G.O.) is thankful to the members of the ITEP Theoretical Physics Department for their hospitality. This investigation was supported in part by the International Science Foundation Grants M9H000 and RYE000 and by the International Association for the Promotion of Cooperation with Scientists from Independent States of the Former Soviet Union Grant INTAS-93-283. 


\section{References}

[1] A.S.Gorski, B.L.Ioffe, A.Yu.Khodjamirian, A.G.Oganesian Z.Phys. C44 (1989) 523; ZhETF 97 (1990) 47.

[2] B.L.Ioffe, Pisma ZhETF 42 (1985) 266, 43 (1986) 316.

[3] V.M.Belyaev, B.L.Ioffe, Nucl.Phys. B310 (1988) 548.

[4] V.M.Budnev, I.F.Ginsburg, G.V.Meledin, V.G.Serbo, Phys.Pep. 15 (1975) 181.

[5] C.Peterson, C.H.Walsh, P.M.Zerwas, Nucl.Phys. B174 (1980) 424.

[6] H.Kolanovski - Two photon physics of $e^{+} e^{-}$storage rings, - Springer, Berlin, Heidelberg, New York 1984.

[7] Ch.Berger, W.Wagner Phys.Rep. 146 (1987) 1.

[8] A.Levy, Report at International Workshop on Deep Inelastic Scattering, Eilat, Israel, February, 1994.

[9] V.A.Novikov, M.A.Shifman, A.I.Vanstein, V.I.Zakharov, Ann.Phys. 105 (1977) 276.

[10] E.Witten, Nucl.Phys. B120(1977) 189.

[11] M.Clück, E.Reya, Phys.Rev D28 (1983) 2749.

[12] W.A.Bardeen, A.J.Buras Phys.Rev. D20 (1979) 166; D21, 2041(E), 1980.

[13] W.Furmanski, R.Petronzio Phys.Lett. 97B (1980) 437.

[14] J.De Witt, L.M.Jones, J.D.Sullivan, D.E.Willen and W.Wyld Phys.Rev.D19 (1979) 2046.

[15] G.Rossi Phys.Rev. D29 (1984) 852.

[16] M.Clück, E.Reya, A.Vogt Phys.Rev. D45 (1992) 3986.

[17] T.Uematsu, T.F.Walsh Nucl.Phys.B199 (1982) 93.

[18] M.Glück, E.Reya, A.Vogt Phys.Rev.D46 (1992) 1973.

[19] M.Glück, E.Reya, M.Stratman, DO-TM 94/14. 
[20] K.Hagiwara, M.Tanaka, I.Watanabe, KEK-TH-376.

[21] R.T.Herrod, W.Wada, B.R.Webber Z.Phys.C9 (1981) 351.

[22] M.Dress, K.Grassie Z.Phys. C28 (1985), 451.

[23] H.Abramowicz, K.Grahula, A.Levy, Phys.Lett. B269 (1991) 451.

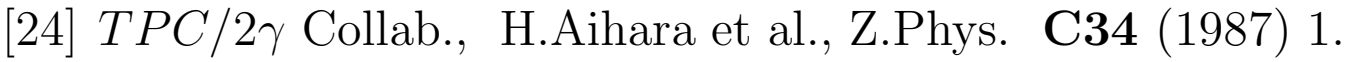

[25] TPC/2 $\gamma$ Collab., J.S.Sterman, UCLA preprint UCLA-HEP-88-004 (1988).

[26] PLUTO Collab. Ch.Berger et al., Z.Phys. C26 (1984) 353.

[27] PLUTO Collab., Ch.Berger et al. Phys.Lett. 142B (1984) 111.

[28] PLUTO Collab., Ch.Berger et al. Nucl.Phys. B281 (1987) 365.

[29] OPAL Collab., R.Akers et al., Z.Phys. C61 (1994) 199-208. 


\section{$\underline{\text { Figure Captions }}$}

Fig. 1. Bare Diagram.

Fig. 2. Hadronic contribution into structure function.

Fig. 3. Some diagrams corresponding to quark and gluon direct production.

Fig. 4. Gluon distribution in the real photon (thick solid line); thin solid line and dotted line - respectively, the contributions from hadronic part and from gluonic condensate, the latter taken with opposite sign. The results are given for $Q^{2}=5,10,50 \mathrm{GeV}^{2}$. The boundary conditions eq.s $(17,18)$ - I variant, correspond to the normalization point $Q_{0}^{2}=1 \mathrm{GeV}^{2}$. The dashed line stands for the result of ref.[16].

Fig. 5. Gluon distribution for virtually polarized photon $\left(p^{2}=\right.$ $-0.5 \mathrm{GeV}^{2}, Q_{0}^{2}=1 \mathrm{GeV}^{2}$ ); thick and dashed lines, respectively - transversally polarized and unpolarized virtual photon case, hadronic part and module of gluon condensate contribution are shown by dashed and dotted lines, respectively.

Fig. 6. The same as Fig.5 for $p^{2}=-1 G e V^{2} ; Q_{0}^{2}=$ $4 \mathrm{GeV}^{2} ; Q^{2}=50 \mathrm{GeV}^{2}$.

Fig. 7. The same as Fig.5 for $p^{2}=-2 G e V_{0}^{2} ; Q_{0}^{2}=$ $6 \mathrm{GeV}^{2} ; Q^{2}=100 \mathrm{GeV}^{2}$.

Fig. 8. Gluon distribution function in the real photon at $Q^{2}=$ $10 \mathrm{GeV}^{2}$ and different $Q_{0}^{2}, Q_{0}^{2}=1 \mathrm{GeV}^{2}$ - thick solid line, $Q_{0}^{2}=2$ - thin solid line). a) Boundary conditions eqs. $(17,18)$ - I variant. b) Boundary conditions eqs. $(18,24)$ - II variant. 
Fig. 9. Gluon distribution in the real photon for the case of the boundary condition (24) and (18) for quarks - II variant of the boundary conditions, $Q_{0}^{2}=1 \mathrm{GeV}^{2}$. The notations are the same as in Fig.4.

Fig. 10 The same as Fig.5 for the II variant (eqs.18,24). The results of [19] are shown by the crossed line for comparison.

Fig. 11 The same as Fig.6 for the II variant (eqs.18,24). The results of [19] are shown by the crossed line for comparison.

Fig.12 The same as Fig.7 for the II variant (eqs.18,24).

Fig. 13 Gluon distribution in the real photon, $Q^{2}=10 \mathrm{GeV}^{2}$. Thick solid line-our results (II variant), thin solid lineour results (I variant), dotted line - the results of [18], dashed line - the results of [22], crossed line - the results of [23].

Fig. 14 Gluon distribution in the real photon; thick solid line our results, thin solid line, dotted and dashed lines - the results of fit [20].

Fig. 15 Structure function $F_{2}$ of real photon for three flavours (thick solid line) at $Q_{0}^{2}=1 \mathrm{GeV}^{2}$ for $Q^{2}=5 \div 15 \mathrm{GeV}^{2}$. Thin solid line and dotted line - contribution from hadron part and gluon condensate, the later - with opposite sign. Experimental data (Refs.[24-29]) are shown for comparison. Also results of Ref.[18,20] are shown by dashed and crossed lines respectively. (On Fig.15a results of PLUTO collab. are at $5.3 \mathrm{GeV}^{2}$ and TPC - at $5.1 \mathrm{GeV}^{2}$.)

Fig. 16 The same Fig.15, for $Q^{2}=20,45 G e V^{2}$. 
Fig. 17 Structure function of transversally polarized virtual photon (thick solid line) and unpolarized virtual photon (dashed line). Thin solid line and dotted line - as in Fig.15. $p^{2}=-0.35 \mathrm{GeV}^{2}, Q_{0}^{2}=1 \mathrm{GeV}^{2}$.

Fig. 18 The same as Fig. 17 for $p^{2}=-1 G e V^{2}, \quad-2 G e V^{2}$ and $Q_{0}^{2}=2 \mathrm{GeV}^{2}, 4 \mathrm{GeV}^{2}$ respectively. Cross line - the results of Ref.[19].

Fig. 19 Comparison of our results in $\overline{M S}$ (thin solid line) and $D I S_{\gamma}$ (thick solid line) schemes for gluon distribution and structure function in real photon case. 
This figure "fig1-1.png" is available in "png" format from: http://arxiv.org/ps/hep-ph/9502412v1 
This figure "fig2-1.png" is available in "png" format from: http://arxiv.org/ps/hep-ph/9502412v1 
This figure "fig3-1.png" is available in "png" format from: http://arxiv.org/ps/hep-ph/9502412v1 
This figure "fig1-2.png" is available in "png" format from: http://arxiv.org/ps/hep-ph/9502412v1 
This figure "fig2-2.png" is available in "png" format from: http://arxiv.org/ps/hep-ph/9502412v1 
This figure "fig3-2.png" is available in "png" format from: http://arxiv.org/ps/hep-ph/9502412v1 
This figure "fig1-3.png" is available in "png" format from: http://arxiv.org/ps/hep-ph/9502412v1 
This figure "fig2-3.png" is available in "png" format from: http://arxiv.org/ps/hep-ph/9502412v1 
This figure "fig3-3.png" is available in "png" format from: http://arxiv.org/ps/hep-ph/9502412v1 